\title{
Short communication: Survey of hepatic copper concentrations in Midwest dairy cows
}

\author{
Jaimie M. Strickland, ${ }^{1,2,3}$ Thomas H. Herdt, ${ }^{2}$ Dodd G. Sledge, ${ }^{3}$ and John P. Buchweitz ${ }^{3 *}$ \\ ${ }^{1}$ Comparative Medicine and Integrative Biology, College of Veterinary Medicine, Michigan State University, East Lansing 48824 \\ ${ }^{2}$ Department of Large Animal Clinical Sciences, College of Veterinary Medicine, Michigan State University, East Lansing 48824 \\ ${ }^{3}$ Department of Pathobiology and Diagnostic Investigation, College of Veterinary Medicine, Michigan State University, East Lansing 48824
}

\section{ABSTRACT}

Previous research from our laboratory and others indicates that liver copper concentrations in dairy cattle are commonly well above those recognized as adequate for the nutritional needs of the animal. It has also been speculated that hepatic copper concentrations have been increasing in recent years. Unlike other species, the threshold at which elevated liver copper concentrations becomes deleterious to hepatocytes is not known for cattle. Therefore, the objectives of this study were 3-fold: (1) to delineate differences in the range and mean dry matter hepatic copper concentration for dairy cattle in a retrospective analysis (January 1, 2007, to December 31, 2015), (2) to investigate hepatic copper concentrations in Midwest cull dairy cattle, and (3) to evaluate histologic changes in hepatocellular morphology in the context of copper concentration in cull cows. Furthermore, microscopic changes in hepatocellular morphology or architecture were examined and scored for evidence of inflammation, fibrosis, necrosis, and abundance of rhodanine-stained granules using hematoxylin and eosin and rhodanine staining. The retrospective analysis found copper concentrations within a range of 3 to $1,963 \mu \mathrm{g} / \mathrm{g}$, with a mean of 473 $\mu \mathrm{g} / \mathrm{g}$. Hepatic copper concentrations in our retrospective study did not increase with time. In our abattoir analysis, copper concentrations ranged from 15 to 978 $\mu \mathrm{g} / \mathrm{g}$, with a mean of $390 \mu \mathrm{g} / \mathrm{g}$. This study found that the range and mean hepatic copper concentrations were comparatively less in the current abattoir study than copper concentrations in our retrospective analysis. There was no evidence for hepatocellular changes associated with increased copper burdens in this study population.

Key words: dairy cow, copper, nutrition

Received August 17, 2018.

Accepted December 20, 2018

*Corresponding author: buchwei2@msu.edu

\section{Short Communication}

Dietary copper is sequestered in the liver of all animals, but liver accumulation can be particularly severe in ruminants due to their limited ability to excrete copper in bile in response to increased intake (Bremner, 1998; López-Alonso et al., 2017). Excess copper can cause hepatocellular damage and liver necrosis. This phenomenon has been well defined in sheep (HumannZiehank et al., 2001; Kupper et al., 2005; Headley et al., 2008; Roubies et al., 2008; Oruc et al., 2009; Lippi Ortolani et al., 2011). In contrast, copper toxicosis in dairy cattle has not been documented as frequently, although reports documenting its occurrence and tabulating hepatic copper concentrations in affected animals are available (Bidewell et al., 2012). The hepatic copper concentrations at which liver injury or toxicosis occurs in cattle are not extensively established and may vary based on physiological state (Bidewell et al., 2012). Additional research examining the potential subclinical effects of hepatic copper concentrations in excess of those necessary for nutritional adequacy is warranted.

The range of bovine hepatic copper concentrations sufficient to support nutritional needs is ill defined, but the expected upper limit has been suggested to lie somewhere between 300 and $500 \mu \mathrm{g} / \mathrm{g}$ of DM (Suttle, 2010; Kendall et al., 2015). Similarly, the hepatic concentration at which copper toxicosis occurs is not precisely defined, although the threshold for increased risk of toxicosis is thought to be greater than $850 \mu \mathrm{g} / \mathrm{g}$ of DM (Suttle, 2016). In addition, the possible health effects caused by hepatic copper concentrations greater than what is nutritionally required but less what causes fulminant toxicosis are also poorly understood.

There are concerns that dairy cattle are being fed excessive supplemental copper to avoid deficiency, and copper toxicities could be a concern for the industry (Lyman, 2013; Grace and Knowles, 2015; Kendall et al., 2015; Lyman et al., 2015). Kendall et al. (2015) reported that the incidence of copper toxicosis has increased in the United Kingdom. Additionally, Grace 
and Knowles (2015) postulated that hepatic copper concentrations in dairy cows have increased generally from 2000 to 2014 in response to oversupplementation in New Zealand.

The National Research Council recommends that lactating dairy cattle consume 14 to $16 \mathrm{mg} / \mathrm{kg}$ copper in the total diet (NRC, 2001). However, this recommendation is affected by the amount of antagonistic minerals present in the feed, such as molybdenum and sulfate. These complex interactions make appropriate copper supplementation challenging for ruminants.

We hypothesized that the range and mean of hepatic copper concentrations in dairy cattle increased from 2007 to 2015. The first objective of this study was to retrospectively evaluate changes in hepatic copper concentrations recorded over time by querying the Michigan State University Veterinary Diagnostic Laboratory (MSU VDL; Lansing, MI) samples that had been submitted from across the United States during the 9-yr period. Clinical histories of the animals tested were unavailable; thus, any inference from the hepatic copper concentrations in this sample to those of the general population of Holstein cattle is inappropriate. However, it seems reasonable to hypothesize that if the copper consumption of cattle increased over time, the time trend would be reflected in these diagnostic samples. The second objective was to survey current hepatic copper concentrations in Midwest dairy cattle by means of an abattoir study of cull cows. The third objective was to evaluate morphologic changes in liver that may be associated with increased copper accumulation. This study was exempted by the Michigan State University Animal Care and Use Committee.

For objective 1, the MSU VDL database was used to retrospectively analyze bovine liver copper concentrations from liver biopsy samples submitted between January 1, 2007, and December 31, 2015. Only adult Holstein cows ( $\geq 2$ yr of age) were included. The database contained trace nutrient mineral concentrations obtained from 782 bovine livers. A total of 340 submissions from 225 owners were included. Submissions contained a mean of 2.3 liver samples per submission (SD $=5.5 ;$ range $=1-80$ samples/submission). Generally, a single sample submission may come from necropsies performed either at the MSU VDL or on farm, and multiple animal submissions were obtained from biopsies in live cows.

For objective 2, an abattoir study was conducted. Liver tissue samples were collected from cull dairy cows in $1 \mathrm{~d}$ at a large abattoir in Western Michigan. Liver samples were collected by a trained abattoir worker from the caudal edge of the right liver lobe. Samples were collected from this area of the liver because it is the portion accessible for liver biopsy in live animals.
Portions of each sample were submersed into formalin within a few hours of collection. The remaining liver was frozen until it could be analyzed for mineral content. For objective 2, liver samples $(\mathrm{n}=149)$ were collected only from adult Holstein dairy cows. However, it is possible that liver was collected from additional dairy breeds as well because hides had been removed before sample collection. All cows included in the study originated from a livestock auction in southwest Illinois. The auction facility reported that most animals sourced from their facility originate from farms within a 100 -mile $(161 \mathrm{~km})$ radius but that they can come from as far as 200 miles $(322 \mathrm{~km})$ away. No dietary or health history could be collected on the study cohort. No samples were taken from carcasses that were condemned. We recognize that the distribution of hepatic copper concentrations in this study cohort may not be representative of the general dairy cattle population, but we speculate that the range of concentrations is likely to be representative of the broader population.

For the measurement of hepatic copper content, 1-g sections of tissue were digested overnight in a $95^{\circ} \mathrm{C}$ oven using approximately 10 times the DM mass of nitric acid. Separate 2-g sections were dried overnight in a $75^{\circ} \mathrm{C}$ oven to determine the DM fraction and calculate dried tissue mass. The digested samples were diluted with water to 100 times the tissue mass. Elemental analysis was performed in accordance with our laboratory procedure first published by Fulton (2016), which uses an inductively coupled plasma mass spectrometer (Agilent Technologies Inc., Santa Clara CA; Wahlen et al., 2005). Briefly, $200 \mu \mathrm{L}$ of each diluted tissue digest and calibration standard was diluted 20 -fold with a solution containing $0.5 \%$ EDTA and Triton X-100, $1 \%$ ammonium hydroxide, $2.0 \%$ propanol, and $5 \mu \mathrm{g} / \mathrm{kg}$ scandium and $7.5 \mu \mathrm{g} / \mathrm{kg}$ germanium, rhodium, indium, and bismuth as internal standards. The inductively coupled plasma mass spectrometer was tuned to yield a minimum sensitivity of 7,500 counts per second for 1 $\mu \mathrm{g} / \mathrm{kg}$ yttrium (mass $=89$ ), less than $1.0 \%$ oxide level as determined by the 156:140 mass ratio, and less than $2.0 \%$ double-charged ions as determined by the 70:140 mass ratio. Elemental concentrations were calibrated using a 5-point linear curve of the analyte:internal standard response ratio. Standards were from Inorganic Ventures (Christainsburg, VA). National Institute of Standards and Technology (Gaithersburg, MD) bovine liver was used as a control. Copper concentrations were reported on a DM basis. Formalin-fixed liver samples from the abattoir study were paraffin embedded and subsequently cut into multiple, and sequential, $5-\mu \mathrm{m}-$ thick sections and stained with hematoxylin and eo$\sin (\mathbf{H} \& \mathbf{E})$ for evaluating morphologic changes and rhodanine to visualize copper storage. All slides were 
reviewed and scored by a board-certified veterinary pathologist (DGS) for the degree of inflammation, fibrosis, and centrilobular hepatocellular change. Additionally, rhodanine-stained slides were evaluated for the relative abundance of copper granules. Rhodaninestained slides were graded on a 0-to- 2 scale, with a score of 0 for no rhodanine-stained granules, a score of 1 for moderate amounts of rhodanine-stained granules in less than $50 \%$ of centrilobular hepatocytes, and a score of 2 for moderate to large numbers of rhodaninestained granules in more than $50 \%$ of the centrilobular hepatocytes.

The existence of a time trend over the years of the retrospective study (objective 1) was assessed by correlating the years and the individual annual means (Spearman, PROC CORR, SAS version 9.4, SAS Institute Inc., Cary, NC). The overall means for part 1 and part 2 were compared by $t$-tests. In part 3 , due to the nonnormality of rhodanine histological scores, differences in median hepatic copper concentration by rhodanine score were assessed by the Kruskal-Wallis test with specific contrasts as calculated by GraphPad Prism version 6 (Dunn, 1964). For all analyses, $P<$ 0.05 was considered significant.

In part 1 , the mean hepatic copper concentration was $473 \mu \mathrm{g} / \mathrm{g}$, with a median of $443 \mu \mathrm{g} / \mathrm{g}$, and concentrations ranged from 3 to $1,963 \mu \mathrm{g} / \mathrm{g}$. All copper concentrations are expressed on a DM basis. From 2007 to 2015 , there was a decreasing trend of hepatic copper concentrations $\left(\mathrm{R}^{2}=0.45, P<0.05\right)$. However, the strength of the time trend association was affected substantially by the mean copper concentration in 2007, which was unusually elevated $(675.87 \mu \mathrm{g} / \mathrm{g})$ relative to the other years. When data from 2007 were removed from the data set, there was no significant change in hepatic copper concentrations across years $\left(\mathrm{R}^{2}=0.013\right.$, $P>0.05)$. These findings contradicted our hypothesis that liver concentrations have been increasing over time, at least within the confines of samples submitted to our laboratory.

Overall, from the 782 cases evaluated for the 9-yr period, $40 \%$ of the MSU VDL liver samples had copper concentrations greater than $500 \mu \mathrm{g} / \mathrm{g}$ of DM and $8 \%$ had concentrations greater than $850 \mu \mathrm{g} / \mathrm{g}$ of DM. This was very similar to the findings of Kendall et al. (2015) for hepatic copper concentrations found in cull Holstein-Friesian cows. Table 1 shows these results stratified by year.

For objective 2, the mean hepatic copper concentration obtained from the abattoir cows was $390 \mu \mathrm{g} / \mathrm{g}$ of $\mathrm{DM}$, with a median of $400 \mu \mathrm{g} / \mathrm{g}$ and a range of 15 to $978 \mu \mathrm{g} / \mathrm{g}$. The mean copper concentration from objective 2 was significantly less than that of objective 1 of our study $(P<0.001)$. In objective $2,26 \%$ of samples

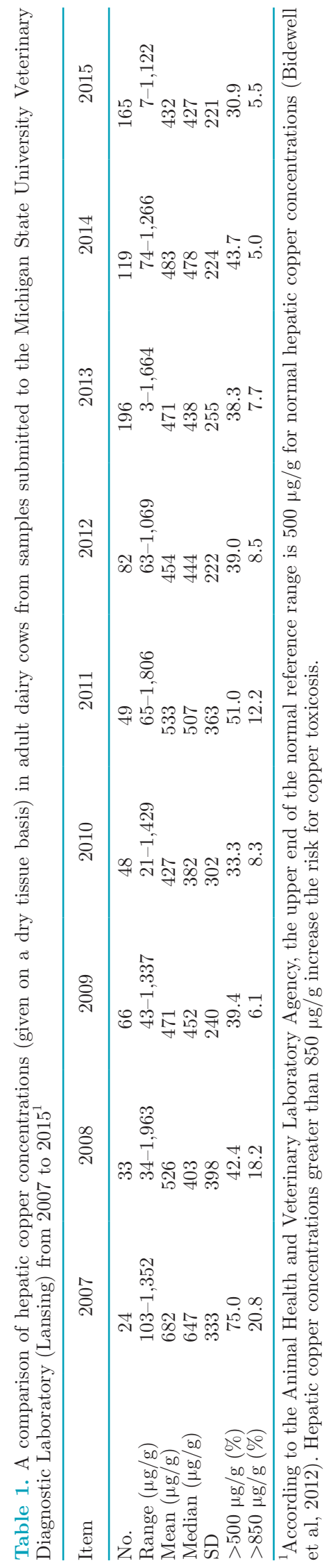

Journal of Dairy Science Vol. 102 No. 5, 2019 


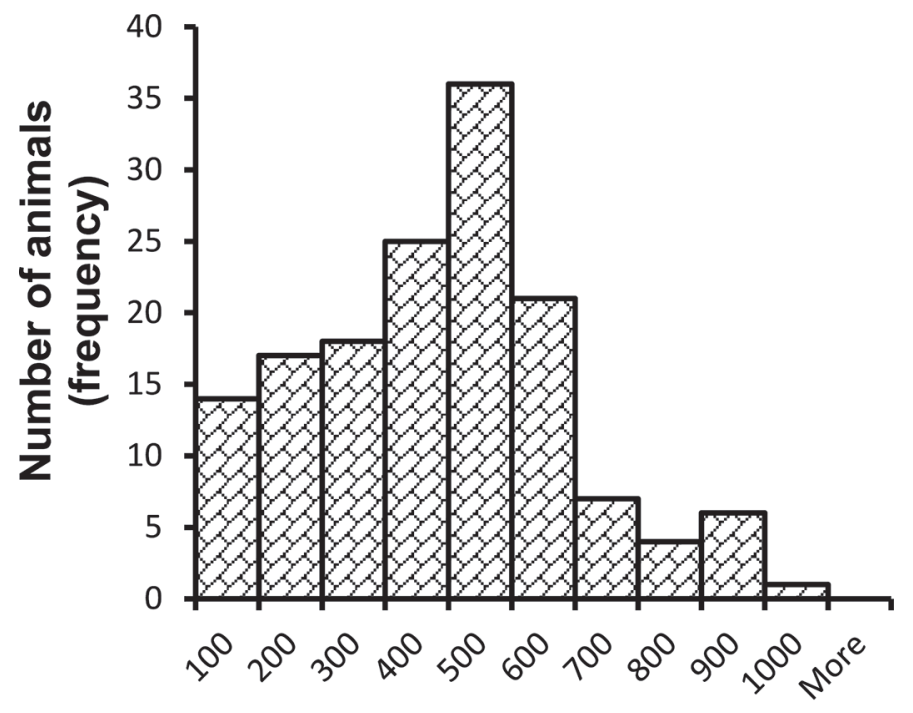

Hepatic copper $(\mu \mathrm{g} / \mathrm{g})$

Figure 1. Frequency distribution of hepatic copper concentrations $(\mu \mathrm{g} / \mathrm{g}$ of DM) from part 2 of the study. Hepatic copper concentrations within a range of 1 to $1,000 \mu \mathrm{g} / \mathrm{g}$ of $\mathrm{DM}$ were tallied and sorted into bins with a width of $100 \mu \mathrm{g} / \mathrm{g}$ of DM. The histogram of the number of animals represented within each bin was left skewed.

had copper concentrations greater than $500 \mu \mathrm{g} / \mathrm{g}$ of DM and $2 \%$ had concentrations greater than $850 \mu \mathrm{g} / \mathrm{g}$ of DM. These results were much lower than the hepatic copper concentrations found in cull Holstein-Friesian cows by Kendall et al. (2015). The frequency distribution of these data can be found in Figure 1.

Last, objective 3 of our study evaluated morphologic changes in the liver associated with the broad distribution of copper concentrations reported in objective 2 . In objective 3 , there was no evidence for centrilobular hepatocellular necrosis, a characteristic finding with copper toxicities, in any of the samples in this study. Furthermore, there was no association between hepatic copper concentrations and centrilobular hepatocellular change of any kind or with degree of hepatic inflammation. There was a significant association between hepatic copper concentrations and fibrosis score; however, fibrosis was generally mild and in the periportal region. There was, however, an expected significant linear relationship between increasing hepatic copper concentration and the relative abundance of rhodaninestained granules as scored by a pathologist $\left(\mathrm{R}^{2}=0.266\right.$, $P<0.01$; Figure 2).

When interpreting mineral analysis results within the context of reference intervals for bovine hepatic copper concentrations, the values are often portrayed as a black-and-white assessment of an animal's copper status. The animal is deemed to be deficient, normal, or supernutritional. For the interpretation of copper status, Suttle (1994) described gray zones at the extremes of typical reference intervals and referred to these as "marginal bands." Briefly, marginal bands span a range of hepatic copper concentrations on either end of a range that would be considered typical for healthy cattle. The marginally deficient bands exist at the low end of the range, nearing deficiency (approximately 6.4-19.2 $\mu \mathrm{g} / \mathrm{g}$ DM of copper). Conversely, the marginally toxic band is at the high end of the range (approximately 340-850 $\mu \mathrm{g} / \mathrm{g}$ DM of copper). The bands represent a range of hepatic copper concentrations over which we would expect to see clinical changes if copper intake was altered. When animals in a herd have hepatic copper concentrations that trend toward the lower concentration of the deficiency band, improvement in herd health and production would be expected by increasing copper supplementation (Suttle, 2010). Conversely, animals with hepatic copper concentrations that trend toward the upper end of the supernutritional marginal band may be at increased risk for copper toxicosis (Suttle, 2016). Some evidence suggests that supernutritional concentrations of hepatic copper put cattle at risk for decreased production and increased disease (LópezAlonso et al., 2006; Suttle, 2010; Bidewell et al., 2012). Sixty percent of the animals from part 2 of this study

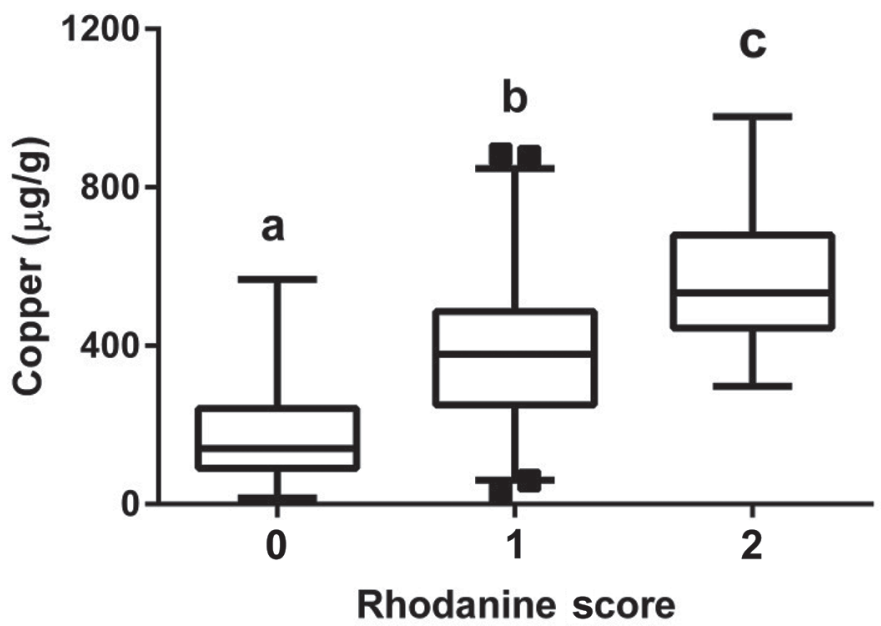

Figure 2. Box-whisker plots of hepatic copper concentrations $(\mu \mathrm{g} / \mathrm{g}$ of $\mathrm{DM})$ by rhodanine staining score. Each box presents the interquartile range, with the midline representing the median copper concentration associated with the designated rhodanine score. Whiskers represent the 2.5 to 97.5 percentiles. A score of 0 indicated no rhodanine-stained granules, a score of 1 indicated moderate amounts of rhodanine-stained granules in less than $50 \%$ of centrilobular hepatocytes, and a score of 2 indicated moderate to large numbers of rhodanine-stained granules in more than $50 \%$ of the centrilobular hepatocytes. Whiskers represent the 2.5 to 97.5 percentiles with black boxes above and below signifying outliers. Letters $(\mathrm{a}-\mathrm{c})$ denote differences in median values $(P<0.001)$. 
had hepatic copper concentrations greater than what is nutritionally required, or supernutritional, suggesting that these animals may have been at increased risk for poor health. Of those, $2 \%$ were above the upper end of the marginally toxic band, putting them at increased risk for clinical copper toxicosis.

In part 3, evidence for supernutritional hepatic copper concentrations was demonstrated by the correlation of rhodanine-stained section scores with hepatic copper concentrations $(P<0.01)$. Copper has a high pro-oxidant potential and is overwhelmingly protein bound once absorbed from the gastrointestinal tract (Dabrowiak, 2017). Copper remains bound to metallothionein, chaperone proteins, and enzymes such as superoxide dismutase once inside the cell (RoatMalone, 2007). Metallothionein is the major protein in cells that functions to store and regulate intercellular copper due to its cysteine-rich structure, which does not allow opportunity for free copper atoms to occur (Sutherland and Stillman, 2011). When hepatocellular copper concentrations reach a critical threshold and normal storage mechanisms are overwhelmed, copper is taken up in lysosomes to prevent oxidative damage in the cell (Goldfischer and Moskal, 1966; Goldfischer and Sternlieb, 1968). It is these copper-filled lysosomes that can be visualized by rhodanine staining (Haratake et al., 1987). Therefore, the presence of these rhodanine-stained granules suggests that normal copper storage mechanisms have been inundated and that hepatic copper concentrations are in excess. It is not well understood whether or how copper contained in lysosomes can be utilized (Polishchuk and Polishchuk, 2016). However, it is likely that once rhodanine-stained granules are visible, additional copper supplementation would not further benefit animal health.

Excessive copper accumulation is characterized by various histological lesions in other species, such as hepatic necrosis in sheep and fibrosis and inflammation in the dog (Humann-Ziehank et al., 2001; Smedley et al., 2009). Special attention was focused on centrilobular zones in this study because copper toxicity specifically causes necrosis in that portion of the lobule in ruminants (Bozynski et al., 2009). In this study, none of the $\mathrm{H} \& \mathrm{E}$ sections demonstrated centrilobular hepatocellular change, such as necrosis, even though $60 \%$ of samples had copper concentrations that were supernutritional. Even with the lack of histological evidence, many researchers would have considered these concentrations dangerously close to toxicosis (Lyman, 2013; Suttle, 2016). Unlike in dogs, no correlation was found between hepatitis and copper concentration. Although many of the H\&E sections examined had some demonstrable inflammation, it was speculated that sections with more severe inflammation might be attributed to causes other than copper accumulation.

In conclusion, this study demonstrated that adult Holstein-Friesian cattle of US origin had a broad range of hepatic copper concentrations, including concentrations in excess of what would be expected for wellnourished cattle. Despite these supernutritional hepatic copper concentrations, there were no histologically visible lesions in the architecture of bovine hepatocytes that could be attributed to copper. More research is needed to investigate the effects of hepatic copper concentrations on other measures of liver damage and function as well as animal health and production.

\section{ACKNOWLEDGMENTS}

The authors thank Micronutrients (Indianapolis, IN) for their support of tissue mineral analysis in this study. The authors also thank C. H. (Hobie) and Carol T. Larson for their generous support through the Michigan State University (East Lansing) College of Veterinary Medicine Endowed Research Funds.

\section{REFERENCES}

Bidewell, C. A., J. R. Drew, J. H. Payne, A. R. Sayers, R. J. Higgins, and C. T. Livesey. 2012. Case study of copper poisoning in a British dairy herd. Vet. Rec. 170:464.

Bozynski, C. C., T. J. Evans, Y. K. Dae, G. C. Johnson, J. M. HughesHanks, W. J. Mitchell, G. E. Rottinghaus, J. Perry, and J. R. Middleton. 2009. Copper toxicosis with hemolysis and hemoglobinuric nephrosis in three adult Boer goats. J. Vet. Diagn. Invest. 21:395-400.

Bremner, I. 1998. Manifestations of copper excess. Am. J. Clin. Nutr. 67:1069S-1073S.

Dabrowiak, J. C. 2017. Metals in Medicine. Wiley, Hoboken, NJ.

Dunn, O. J. 1964. Multiple comparisons using rank sums. Technometrics $6: 241-252$.

Fulton, J. 2016. The effect of copper and zinc source on pre-weaning performance of cows, health and performance of suckling calves, and post-weaning feedlot performance, carcass composition, and meat quality of calves. MS Thesis. South Dakota State University, Brookings.

Goldfischer, S., and J. Moskal. 1966. Electron probe microanalysis of liver in Wilson's disease. Simultaneous assay for copper and for lead deposited by acid phosphatase activity in lysosomes. Am. J. Pathol. 48:305-315.

Goldfischer, S., and I. Sternlieb. 1968. Changes in the distribution of hepatic copper in relation to the progression of Wilson's disease (hepatolenticular degeneration). Am. J. Pathol. 53:883-901.

Grace, N., and S. Knowles. 2015. Taking action to reduce the risk of copper toxicity in cattle. Vet. Rec. 177:490-491.

Haratake, J., A. Horie, and S. Takeda. 1987. Histochemical and ultrastructural study of copper-binding protein in hepatocellular carcinoma. Cancer 60:1269-1274.

Headley, S. A., A. C. F. dos Reis, T. L. D. R. Nakagawa, and A. P. F. R. L. Bracarense. 2008. Chronic copper poisoning in sheep from Northern Paraná, Brazil: Case report. Semin. Cienc. Agrar. 29:179-184.

Humann-Ziehank, E., M. Coenen, M. Ganter, and K. Bickhardt. 2001. Long-term observation of subclinical chronic copper poisoning 
in two sheep breeds. J. Vet. Med. A Physiol. Pathol. Clin. Med. 48:429-439.

Kendall, N. R., H. R. Holmes-Pavord, P. A. Bone, E. L. Ander, and S. D. Young. 2015. Liver copper concentrations in cull cattle in the UK: Are cattle being copper loaded? Vet. Rec. 177:493.

Kupper, J., A. Bidaut, A. Waldvogel, B. Emmenegger, and H. Naegeli. 2005. Treatment of chronic copper poisoning with oral application of ammonium molybdate and natrium sulphate in milk sheep. Schweiz. Arch. Tierheilkd. 147:221-226.

Lippi Ortolani, E., C. H. Machado, A. H. Hamad Minervino, R. Alves Barreto-Junior, C. Satsuki Mori, and S. Arlington Headley. 2011. Clinical observations and acid-base imbalances in sheep during chronic copper poisoning. Semin. Cienc. Agrar. 32:1123-1132.

López-Alonso, M., P. Carbajales, M. Miranda, and V. Pereira. 2017. Subcellular distribution of hepatic copper in beef cattle receiving high copper supplementation. J. Trace Elem. Med. Biol. 42:111116.

López-Alonso, M., A. Crespo, M. Miranda, C. Castillo, J. Hernández, and J. L. Benedito. 2006. Assessment of some blood parameters as potential markers of hepatic copper accumulation in cattle. J. Vet. Diagn. Invest. 18:71-75.

Lyman, D. 2013. Copper accumulation in Wisconsin Holsteins. Accessed Feb. 27, 2018. http://www.wvdl.wisc.edu/index.php/copper -accumulation-wisconsin-holsteins/.

Lyman, D., L. J. Clark, and K. Campbell. 2015. Copper accumulation in Wisconsin Holsteins with indications of oxidative liver damage. J. Vet. Med. Res. 2:1021.
NRC (National Research Council). 2001. Nutrient Requirements of Dairy Cattle. 7th rev. ed. National Academies Press, Washington, DC.

Oruc, H. H., M. Cengiz, and A. Beskaya. 2009. Chronic copper toxicosis in sheep following the use of copper sulfate as a fungicide on fruit trees. J. Vet. Diagn. Invest. 21:540-543.

Polishchuk, E. V., and R. S. Polishchuk. 2016. The emerging role of lysosomes in copper homeostasis. Metallomics 8:853-862.

Roat-Malone, R. M. 2007. Bioinorganic Chemistry: A Short Course. Wiley, Hoboken, NJ.

Roubies, N., N. D. Giadinis, Z. Polizopoulou, and S. Argiroudis. 2008. A retrospective study of chronic copper poisoning in 79 sheep flocks in Greece (1987-2007). J. Vet. Pharmacol. Ther. 31:181-183.

Smedley, R., T. Mullaney, and W. Rumbeiha. 2009. Copper-associated hepatitis in Labrador Retrievers. Vet. Pathol. 46:484-490.

Sutherland, D. E. K., and M. J. Stillman. 2011. The "magic numbers" of metallothionein. Metallomics 3:444-463.

Suttle, N. F. 1994. Meeting the copper requirements of ruminants. Recent Devel. Ruminant Nutr. 4:221-236.

Suttle, N. F. 2010. Mineral Nutrition of Livestock. CAB International, Wallingford, UK.

Suttle, N. F. 2016. Reducing the risk of copper toxicity in dairy cattle. Vet. Rec. 178:196.

Wahlen, R., L. Evans, J. Turner, and R. Hearn. 2005. The use of collision/reaction cell ICP-MS for the determination of elements in blood and serum samples. Spectroscopy 20:84-89. 